\title{
Many-body effects in photoluminescence of heavily doped AlGaAs/InGaAs/GaAs heterostructures
}

\author{
Z.Ya. Zhuchenko, G.G. Tarasov, S.R.Lavorik, Yu.I.Mazur, M.Ya.Valakh, \\ H.Kissel*, W.T.Masselink*, U.Mueller, C. Walther* \\ Institute of Semiconductor Physics, NAS of Ukraine, \\ 45, prospect Nauki, 03650 Kiev, Ukraine \\ * Department of Physics, Humboldt-Universitat zu Berlin, Invalidenstrasse 110, D-10115 Berlin, Germany
}

\begin{abstract}
A photoluminescence (PL) study of pseudomorphic modulation-doped $\mathrm{Al}_{\mathrm{x}} \mathrm{Ga}_{1-\mathrm{x}} \mathrm{As} / \mathrm{In}_{\mathrm{y}}$ $\mathrm{Ga}_{1-\mathrm{y}} \mathrm{As} / \mathrm{GaAs}$ heterostructures possessing high electron density shows a fundamental change of the PL spectrum under excitation density increase. In its high energy tail the PL peak undergoes principal transformations caused by repelling the Fermi-edge singularity (FES) and excitonic states. The character of repelling depends crucially on the excitation density and temperature. At low temperatures an appearance of the FES feature has been observed for the first time under excitation density elevation. This appearance is accompanied by formation of an abrupt high energy edge and occurs far below by intensity the hybridized $n=2$ exciton manifestation. Strong screening of the $n=2$ exciton state by photoexcited carriers is observed. The PL behavior under excitation density increase and temperature elevation near the Fermi edge is explained in terms of strong carrier density effect on the FES manifestation and is referred to the two-dimensional (2D) electron gas properties not yet explored theoretically.
\end{abstract}

Keywords: heterostructure, quantum well, photoluminescence, Fermi-edge singularity, Fano interference.

Paper received 18.10.99; revised manuscript received 14.12.99; accepted for publication 17.12.99.

\section{Introduction}

Pseudomorphic modulation-doped $\mathrm{Al}_{\mathrm{x}} \mathrm{Ga}_{1-\mathrm{x}} \mathrm{As} / \mathrm{In}_{\mathrm{y}} \mathrm{Ga}_{1-\mathrm{y}} \mathrm{As} /$ GaAs heterostructures provide nearly ideal conditions for the study of many-body effects, namely, Fermi-edge singularity (FES), band-gap renormalization, or shake-up processes [1-4]. Due to high density of the two-dimensional electron gas (2DEG) in these systems, the correlation effects are of great importance resulting in the rearrangement of electron and hole subsystems in order to reduce the Coulombic interaction. It has been shown theoretically that two principal conditions must be satisfied in order to observe the FES: i) the strong overlap of wave functions for spatially separated electron and hole to ensure the effective electron-hole correlation, ii) the abrupt Fermi edge $[5,6]$. However, it has been also demonstrated recently that these conditions are necessary but, likely, not sufficient for such an observation [7]. We report here on the principally new behavior of FES providing the strong support of the latter assumption.

Typically the FES is identified: (i) in photoluminescence (PL) as an enhancement of the oscillator strength for transitions close to the Fermi edge when the disorder or hole localization violate the momentum conservation law and al- low the transitions nominally forbidden by this law; (ii) in absorption it appears as an excitonic enhancement of a factor $\sim 2$ at $4 \mathrm{~K}$ [8]. FES related effects demonstrate strong temperature, excitation density and magnetic field dependencies. Chen et al. [9] observed for $\mathrm{Al}_{\mathrm{x}} \mathrm{Ga}_{1-\mathrm{x}} \mathrm{As} / \mathrm{In}_{\mathrm{y}} \mathrm{Ga}_{1-\mathrm{y}} \mathrm{As} /$ GaAs in perpendicular magnetic field $B$ very strong periodic in $B^{-1}$ intensity oscillations for the PL at low temperature caused by the resonant enhancement of the FES in the highest occupied Landau level. The electron scattering by holes becomes substantially enhanced due to resonance with excitons of the $n=2$ empty subband. The behavior of the FES and the $n=2$ exciton under near-resonance condition between the $n=2$ electronic subband and the Fermi energy $E_{F}$ has been recently studied in $\mathrm{Al}_{\mathrm{x}} \mathrm{Ga}_{1-\mathrm{x}} \mathrm{As} / \mathrm{In}_{\mathrm{y}} \mathrm{Ga}_{1-\mathrm{y}} \mathrm{As} / \mathrm{GaAs}$ by means of $\mathrm{PL}$ and PL excitation spectra under temperature and excitation density variation [10]. It has been shown that the FES quenches rapidly with a temperature and/or excitation density increase whereas the magnitude of the $n=2$ exciton feature grows accompanied by a blue shift of the energetic position. In all previous studies the FES feature either definitely exists or definitely does not at low temperatures. If existing, its further evolution is traced under temperature, magnetic field or excitation density variation. We demonstrate here for the first time the low temperature appearance of the FES in the PL spectra, although it was absent initially. 


\section{Z.Ya. Zhuchenko et al.: Many-body effects in photoluminescence of heavily doped...}

\section{Experimental details}

Pseudomorphic modulation-doped $\mathrm{Al}_{\mathrm{x}} \mathrm{Ga}_{1-\mathrm{x}} \mathrm{As} / \mathrm{In}_{\mathrm{y}} \mathrm{Ga}_{1-\mathrm{y}} \mathrm{As} /$ GaAs heterostructures were specially grown to meet the conditions of the FES observation. The typical epilayer sequence consists of a GaAs buffer layer $(d=80 \mathrm{~nm})$, an undoped $\mathrm{In}_{\mathrm{y}} \mathrm{Ga}_{1-\mathrm{y}} \mathrm{As}$ strained quantum well (QW) (2DEG channel) $(y=0.19$, QW thickness $D=15 \mathrm{~nm})$, an $\mathrm{Al}_{\mathrm{x}} \mathrm{Ga}_{1-\mathrm{x}} \mathrm{As}$ undoped spacer $(x=0.2, d=7.5 \mathrm{~nm})$, an $\mathrm{Al}_{\mathrm{x}} \mathrm{Ga}_{1-\mathrm{x}}$ As heavily Sidoped supplier layer $\left(x=0.2, d=35 \mathrm{~nm}, N_{D}=2 \cdot 10^{18} \mathrm{~cm}^{-3}\right)$ and a Si-doped GaAs cap layer $\left(d=5 \mathrm{~nm}, N_{D}=2.5 \cdot 10^{18} \mathrm{~cm}^{-3}\right)$. The electron sheet density $N_{s}$ of the $2 \mathrm{D}$ electrons, obtained from the low-field Hall measurements down to $4.2 \mathrm{~K}$ is $N_{s}=$ $1.18 \cdot 10^{12} \mathrm{~cm}^{-2}$. Double-crystal X-ray diffraction and simulation of the double X-ray rocking curve were used to verify the samples' structural parameters. The X-ray patterns indicated a high quality of the structures under investigation. The PL was excited by the 514.5-nm line of a cw $\mathrm{Ar}^{+}$laser. The PL radiation was dispersed through a 3/4-m CzernyTurner scanning spectrometer, with a spectral resolution better than $0.1 \mathrm{meV}$.

\section{Results and discussion}

In order to be more precise in further assignment we calculate the subband structure for the $\operatorname{In}_{\mathrm{y}} \mathrm{Ga}_{1-\mathrm{y}} \mathrm{As}$ quantum well in our heterostructures. The coupled Schrödinger and Poisson equations were solved self-consistently. The Fermi level for a given temperature was calculated by iteration from the charge-neutrality condition. The subband energies and the energy eigenfunctions for the $\mathrm{In}_{0.19} \mathrm{Ga}_{0.81} \mathrm{As} \mathrm{QW}$ are shown in Fig. 1 . The intersubband separation $\Delta=E_{2}-E_{1}$ is found to be $48 \mathrm{meV}$, and $E_{F}$ is of about $5 \mathrm{meV}$ smaller than the $E_{2}$ energy. Obviously the wave function of the $n=1$ electron state peaks nearby the $\mathrm{Al}_{\mathrm{x}} \mathrm{Ga}_{1-\mathrm{x}} \mathrm{As} / \mathrm{In}_{\mathrm{y}} \mathrm{Ga}_{1-\mathrm{y}} \mathrm{As}$ interface, while the wave function of the $n=2$ electron state has a node, and its main peak is in proximity of the $\operatorname{In}_{\mathrm{y}} \mathrm{Ga}_{1-}$ ${ }_{\mathrm{y}} \mathrm{As} / \mathrm{GaAs}$ interface. The wave functions calculated allow to find the ratio of the electron-hole overlap integrals squared for the $E_{21}$ and $E_{11}$ transitions. It takes the value of 12.7 . Possessing the information derived from calculations, one can expect a single-peaked PL spectrum from the $\mathrm{Al}_{\mathrm{x}} \mathrm{Ga}_{1-\mathrm{x}} \mathrm{As} / \mathrm{In}_{\mathrm{y}} \mathrm{Ga}_{1-\mathrm{y}} \mathrm{As} / \mathrm{GaAs}$ heterostructure under investigation.

Fig. 2a shows a characteristic PL spectrum at $4.2 \mathrm{~K}$ and the evolution of its high energy tail under different excitation intensities (Fig. 2b). The strong feature ( $E_{11}$-transition) at $1.284 \mathrm{eV}$ (Fig. 2a) stems from the recombination of $n=1$ electrons and $n=1$ heavy holes and has a typical asymmetric line shape. The recombination peaks near the $n=1$ subband bottom $(\vec{k}=0)$ and strongly decreases in intensity towards $E_{F}$. Due to impurity presence the momentum conservation is broken and the transitions from electrons in states up to $k=k_{F} \sim 2 \cdot 10^{6} \mathrm{~cm}^{-1}$ become allowed. The shape of spectral feature is well described in terms of the microscopic theory, developed by Lyo and Jones [11] for the steadystate PL at low temperatures in modulation-doped degenerate direct-band-gap semiconductor QW's. The following set

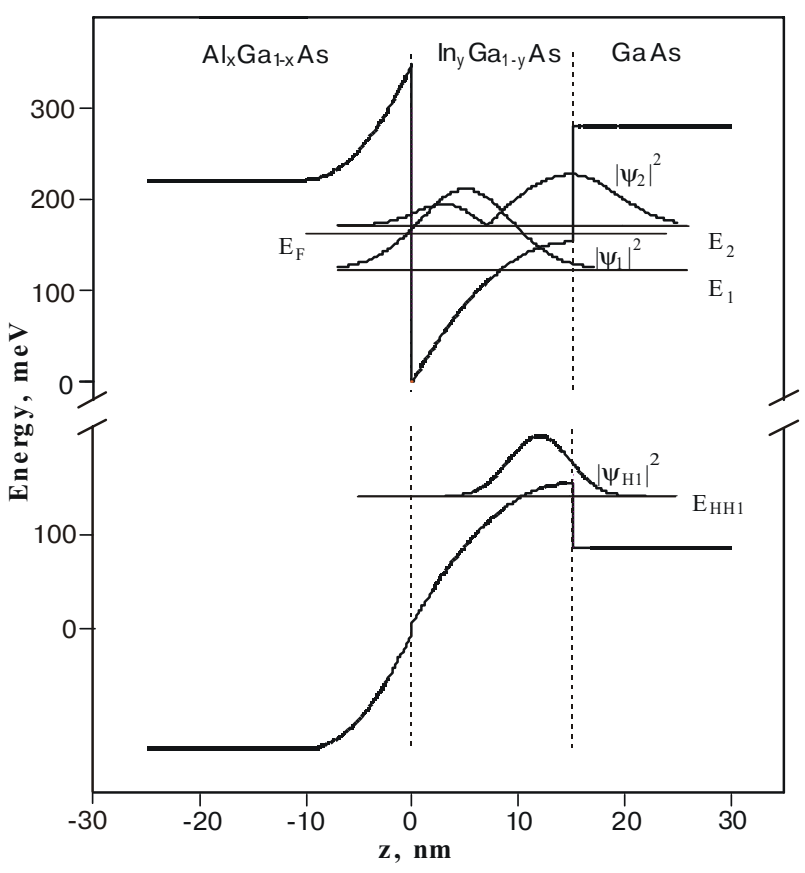

Fig. 1. Conduction and valence band profiles found from a coupled Schrodinger-Poisson analysis for $\mathrm{Al}_{\mathrm{x}} \mathrm{Ga}_{1-\mathrm{x}} \mathrm{As} / \mathrm{In}_{\mathrm{y}} \mathrm{Ga}_{1-\mathrm{y}} \mathrm{As} / \mathrm{GaAs}$ heterostructure. Squared envelope functions for electrons and holes are also shown. Distance is measured from $\mathrm{AlGaAs} / \mathrm{InGaAs}$ interface position.

of parameters was derived from the fit: $E_{g}=1.278 \mathrm{meV}$, $\Gamma=13.8 \mathrm{meV}$ ( $\Gamma$ is the full width at the half maximum), $E_{F}=$ $44 \mathrm{meV}$. The obtained $E_{F}$ value coincides well with that found from the self-consistent solution of Schrodinger and Poisson equations $\left(E_{F}=43 \mathrm{meV}\right)$. The low-energy luminescence threshold on the energy axis (see Fig. 2a) is slightly shifted towards the red side with respect to the $E_{11}$ peak position. This is a consequence of 2D-carrier scattering by the charge impurities strongly depending on the thickness of the spacer layer. These observations convince us that the PL profile is mainly defined by the electron-hole recombination accompanied by impurity and disorder-assisted processes that result in broadening and a blue shift of the PL maximum.

The transformation of the PL line shape in the close vicinity of $E_{F}$ under excitation intensity (Fig. 2b) and temperature variations gives a strong evidence for the FES existence. Taking into account the close proximity of $E_{F}$ and $E_{2}$ energies in our experiment, a strong interaction between the elementary excitations near the Fermi level and the $n=2$ bare (atomic) exciton is expected. We observe new peculiarities of FES manifestation. As one can see from the PL spectra (Fig. 2b) at the modest excitation level, the PL tail spreads far beyond the $E_{F}$ boundary. No traces of the $n=2$ exciton exist. Increasing the excitation density consequently, the FES peculiarity clearly developes with prominent shifting towards the energies below $E_{F}$. The steepness of the spectral dropfall after the FES feature abruptly grows and no PL signal is detected behind $E_{F}$. Further increase of the excitation density gives the hint for the $n=2$ feature appearance $\left(E_{21}\right.$ transition), which moves towards higher energy. Finally, the $E_{11}$ and $E_{21}$ peaks are present only at higher intensities and 


\section{Z.Ya. Zhuchenko et al.: Many-body effects in photoluminescence of heavily doped...}
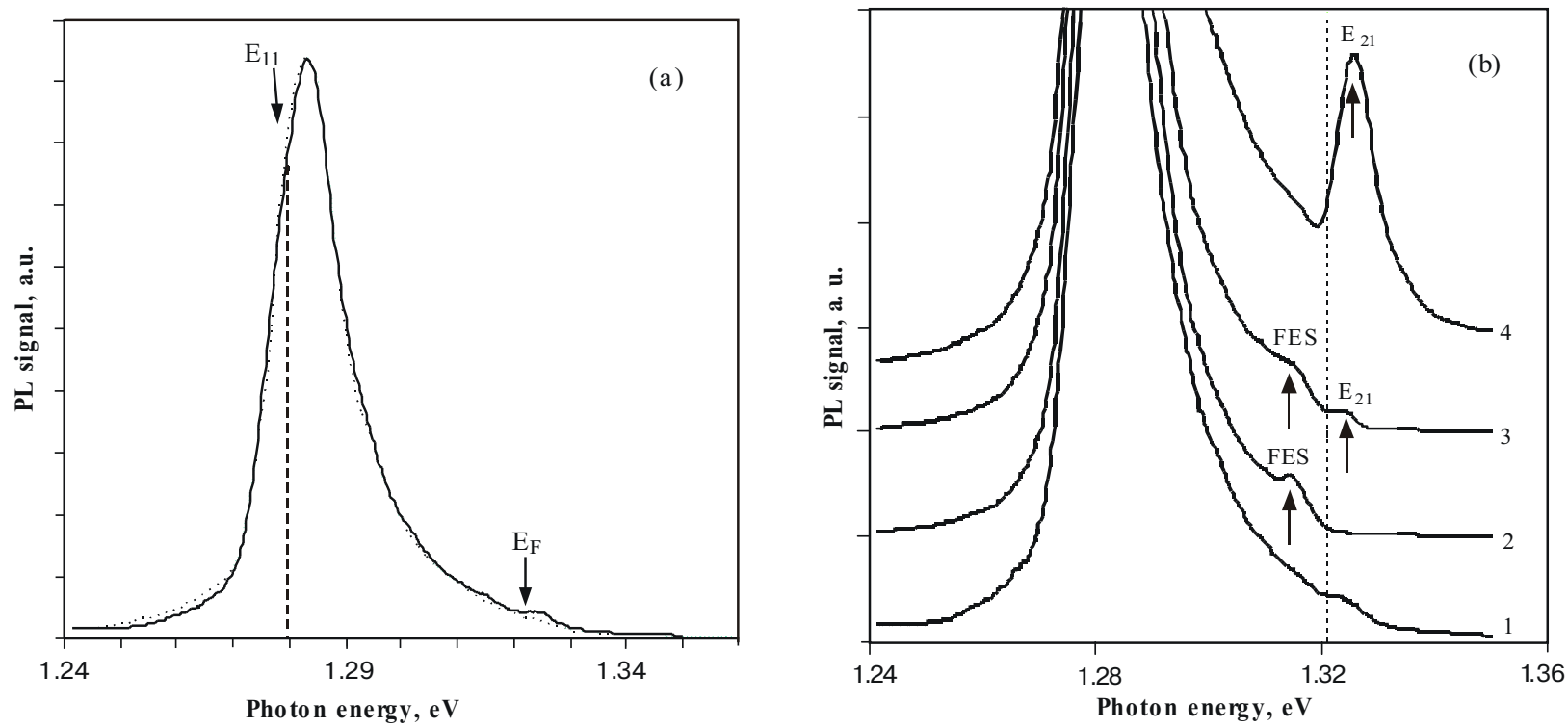

Fig. 2. PL spectrum from $\mathrm{Al}_{0.2} \mathrm{Ga}_{0.8} \mathrm{As} / \mathrm{In}_{0.19} \mathrm{Ga}_{0.81} \mathrm{As} / \mathrm{GaAs}$ heterostructure under various excitation intensity. $T=4.2 \mathrm{~K}$ : a) PL spectrum at lowest excitation power. Dashed line presents the fit in terms of Lyo and Jones theory [11]. Low-energy tail is approximated by Urbach form. Arrows stand for $E_{11}$ and $E_{F}$ energies. b) Transformation of the high energy PL tail under intensity elevation. Curves 1 to 4 correspond to the intensities: $1-0.002 I_{0}, 2-0.01 I_{0}, 3-0.1 I_{0}, 4-I_{0}$, respectively, $\left(I_{0}=10 \mathrm{~W} / \mathrm{cm}^{2}\right)$. Transition energies for the FES and the $n=2$ exciton are shown by arrows up.

the FES decays completely.

The optical oscillator strength for the electrons at the Fermi level annihilating with photoholes becomes enhanced by over two orders of magnitude when the resonant coupling with the excitonic state is switched on [6]. This coupling is described in terms of Fano interference [13]. The Fano resonance aspect of interaction between an exciton and 2D electrons in asymmetrically-doped single $\mathrm{In}_{\mathrm{y}} \mathrm{Ga}_{1-\mathrm{y}} \mathrm{As} \mathrm{QW}$ 's was discussed by Chen et al. [9], without solving the manybody problem. Elaborated theoretical treatment including the band-gap renormalization, static screening, and dynamical response of the Fermi sea has been carried out by Hawrylak [6]. With inclusion of the hole self-energy, the position of the actual emission line falls close to the bare excitonic transition, and in absorption two peaks arise, one related to the bound exciton state. For increasing carrier density the emission spectrum resembles the single-particle density-of-states in the conduction band with enhancement at the Fermi level, while the absorption spectrum evolves into a blueshifted FES. The predicted behavior is somewhat obscuring in comparison with that observed in experiment, including our data and those presented in Ref. [10].

Indeed, at first stage we observed the spread high energy tail of the PL feature (curve 1 in Fig. 2b). At low temperature and low pumping level, it is reasonably described in terms of the electron-heavy hole recombination contributed by impurity and disorder-assisted processes (Fig. 2a). The FES enhancement, if exists, is of a negligible magnitude. Elevating the excitation density in addition to the excitations of the Fermi sea, an increasing contribution from excitations including holes in the $n=1$ heavy hole subband and electrons in the second subband arises. Due to strong reso- nance coupling of excitons with excitations of the Fermi sea, two sets of hybridized states appear, separated by the region of destructive Fano interference. Those related to Fermi edge are shifted by energy towards low energy side, others related to hybridized excitons move to higher energies. With respect to modest excitation level, these latter can not be observed in PL because the $n=2$ electron level is empty under the considered conditions. As a result in PL spectrum (curve 2 in Fig. 2b) we observed typical FES enhancement with abrupt fall-down from high energy side, signalizing about the Fano dip existence. Further increase of excitation density leads to population of the $n=2$ electron level and observation of the $E_{21}$ transition, corresponding to the hybridized excitonic state else (curve 3 in Fig. 2b). Elevating the intensity, we reach the level when both the FES and excitonic emission are subsequently quenched and the free-2D electron-hole recombination becomes dominating at the high-energy PL tail (curve 4 in Fig. 2b).

Fig. 3 shows the motion of the FES and $n=2$ related features under intensity variation. It is clearly seen the region of intensities within the FES originates only. Strong and rapid red shift accompanies the FES appearance. This shift is practically saturated when the hybridized $n=2$ exciton state arises. The $n=2$ related feature demonstrates a pronounced blue shift with a tendency to saturation, reflecting the screening of the $n=2$ exciton by excess electrons. It is noteworthy that the spectral shifts within the $E_{F}$ proximity are not accompanied by any noticeable shift of the $E_{11}$ transition when the excitation level increases. The blueshift of the $E_{21}$ peak is interpreted in terms of transition from the hybridized exciton state to the bare (atomic) exciton [8-10,12], when screening the $n=2$ excition by excess electrons is 
Z.Ya. Zhuchenko et al.: Many-body effects in photoluminescence of heavily doped...

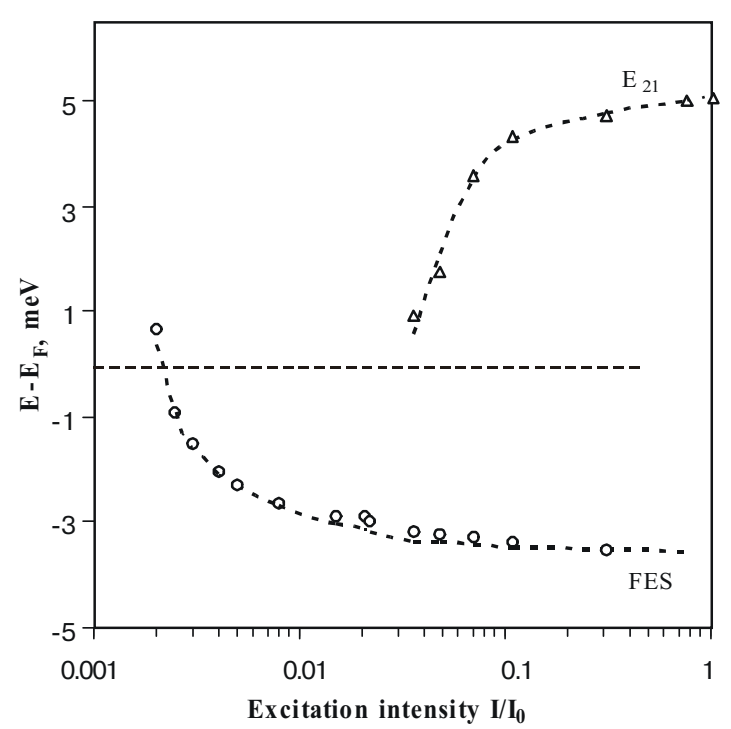

Fig. 3: Shift by energy for the FES and $n=2$ related features in the PL spectrum under excitation power increase.

included. Insensitivity of the transition $E_{11}$ to the pumping density gives evidence that the internal electrical field does not change under intensity increase, and therefore the blueshift saturation for the $E_{21}$ transition is mainly connected with the exciton screening and the transition from the excitonic recom-binaton to the free-electron-hole recombination. In fact we show here that the carrier concentration strongly influences the character of the FES and the $n=2$ exciton manifestation. This is consistent with the conclusion derived from PL investigation in the low-doped $\operatorname{In}_{\mathrm{x}} \mathrm{Ga}_{1-\mathrm{x}} \mathrm{As} / \mathrm{InP}$ heterostructures [7]. It was shown that in spite of the fact that both a close position of the Fermi edge to the empty $n=2$ electron subband and hole localization by alloy fluctuation potential provide the optimal conditions for the occurrence of the FES, it is not sufficient the FES enhancement to be developed, and the carrier concentration has to be adjusted also. We conclude that even modest variation of the concentration, caused by light illumination or heating, can substantially influence the FES development. Indeed, an insignificant eleva-tion of temperature (from $4.2 \mathrm{~K}$ to $15 \mathrm{~K}$ ) gives rise to a substantial broadening and subsequent smearing out the FES related feature in PL. The interpretation given above is consistent with the PL behavior observed, but we are on conscience that other models can be admitted for discussion.

\section{Conclusions}

Summarizing the results of PL study of pseudomorphic modulation-doped $\mathrm{Al}_{\mathrm{x}} \mathrm{Ga}_{1-\mathrm{x}} \mathrm{As} / \mathrm{In}_{\mathrm{y}} \mathrm{Ga}_{1-\mathrm{y}} \mathrm{As} / \mathrm{GaAs}$ heterostructures, possessing the high electron density, we observed a fundamental change of the PL spectrum under excitation density increase. High-energy tail peculiarities are related to the repelling of the FES and excitonic states. The character of repelling depends crucially on the excitation density and temperature. At low temperature, the appearance of the FES feature has been observed for the first time under increasing the excitation density. Pronouncing the FES in PL is accompanied by the formation of an abrupt high energy edge and occurs far below the intensity of the hybridized $n=2$ exciton manifestation. Strong screening of the $n=2$ exciton state by photoexcited carriers is observed, resulting in $2 \mathrm{D}$ electron gas-heavy hole recombination for the second electron subband. A strong excitation power dependence of the 2D-related PL could be the result of the photoinduced change of the potential distribution across the QW structure. This has been previously observed in $\mathrm{Al}_{\mathrm{x}} \mathrm{Ga}_{1-\mathrm{x}} / \mathrm{GaAs}$ quantum structures $[14,15]$, too. A strong band bending can arise from the neutralization of the ionized residual acceptors by photocreated holes in the $\mathrm{In}_{\mathrm{y}} \mathrm{Ga}_{1-}$ yAs channel and the neutralization of the ionized donors in the barriers by photocreated electrons. The reduction of the band bending under illumination leads to a typical blue shift of the PL spectrum. We have not observed a noticeable blue shift of the PL spectrum, and, hence, these reasons have to be ruled out. We incline to explain the behavior of the PL near the Fermi edge by strong carrier density effect on the FES manifestation not yet explored theoretically.

This work is supported by NATO linkage grant.

\section{References}

1. C. Delalande, G. Bastard, J. Orgonasi, J. A. Brum, H. W. Liu, M. Voos, G. Weimann, and W. Schlapp, Many-body effects in a modulation-doped semiconductor quantum well // Phys. Rev. Lett. 59(23), pp. 2690-2692 (1987).

2. M. S. Skolnick, J. M. Rorison, K. J. Nash, D. J. Mowbray, P. R. Tapster, S. J. Bass, and A. D. Pitt, Observation of a many-body edge singularity in quantum-well luminescence spectra // Phys.Rev. Lett. 58(20), pp. 2130-2133 (1987).

3. Y. C. Chang and G. D. Sanders, Band-mixing effect on the emission spectrum of modulation-doped semiconductor quantum wells // Phys. Rev. B 32(8), pp. 5521-5524 (1985).

4. D. Heiman, B. B. Goldberg, A. Pinczuk, C. W. Tu, A. C. Gossard, and J. H. English, Optical anomalies of the two-dimensional electron gas in the extreme magnetic quantum limit// Phys. Rev. Lett. 61(5), pp. 605-608 (1988).

5. T. Uenoyama and L. J. Sham, Many-body theory of magnetooptical spectra in doped quantum wells // Phys. Rev. B 39(15), pp. 11044-11049 (1989).

6. P. Hawrylak, Optical hole in a two-dimensional electron gas // Phys. Rev. B 42(14), pp. 8986-8990 (1990).

7. I.A. Buyanova, T.Lundstrom, A.V. Buyanov, W.M. Chen, W.G. Bi, and C. W. Tu, Strong effects of carrier concentration on the Fermi-edge singularity in modulation-doped $\mathrm{InP} / \mathrm{In}_{\mathrm{x}} \mathrm{Ga}_{1-\mathrm{x}} \mathrm{As}$ heterostructures // Phys. Rev. B 55(11), pp. $7052-7058$ (1997).

8. T. A. Fisher, P. E. Simmonds, M. S. Skolnick, A. D. Martin, and R. S. Smith, Fermi-energy edge singularity and excitonic enhancement associated with the second subband in asymmetric modulation-doped quantum wells // Phys. Rev. B 48(19), pp. 1425314263 (1993).

9. W. Chen, M. Fritze, W. Walecki, and A. V. Nurmikko, Excitonic enhancement of the Fermi-edge singularity in a dense two-dimensional electron gas // Phys. Rev. B 45(15), pp. 8464-8477 (1992).

10. S. J. Xu, S. J. Chua, X. H. Tang, and X. H. Zhang, Strong interaction of Fermi-edge singularity and exciton related to $N=2$ subband in a modulation-doped $\mathrm{Al}_{\mathrm{x}} \mathrm{Ga}_{1-\mathrm{x}} \mathrm{As} / \mathrm{In}_{\mathrm{y}} \mathrm{Ga}_{1-\mathrm{y}} \mathrm{As} / \mathrm{GaAs}$ quantum well // Phys. Rev. B 54(24), pp. 17701-17704 (1996).

11. S. K. Lyo and E. D. Jones, Photoluminescence line shape in degenerate semiconductor quantum wells // Phys. Rev. B 38(6), pp. 4113-4119 (1988). 


\section{Z.Ya. Zhuchenko et al.: Many-body effects in photoluminescence of heavily doped...}

12. C. Colvard, N. Nouri, H. Lee, and D. Ackley, Optical investigations of the high-density electron gas in pseudomorphic $\operatorname{In}_{\mathrm{x}} \mathrm{Ga}_{1}$ ${ }_{x}$ As quantum-well structures // Phys. Rev. B 39(11), pp. 80338036 (1989).

13. U. Fano, Effects of configuration interaction on intensities and phase shifts // Phys. Rev. 124(6), pp. 1866-1878 (1961).

14. I. V. Kukushkin, K. von Klitzing, K. Ploog, and V. B. Timofeev, Radiative recombination of two-dimensional electrons in accep- tor $d$-doped GaAs- $\mathrm{Al}_{\mathrm{x}} \mathrm{Ga}_{1-\mathrm{x}} \mathrm{As}$ single heterojunctions // Phys. Rev. $B$ 40(11), pp. 7788-7792 (1989).

15. Y. R. Yuan, K. Mohammed, M. A. A. Pudensi, and J. L. Merz, Effects of carrier confinement in graded AlGaAs/GaAs heterojunctions // Appl. Phys. Lett. 45(7), pp. 739-741 (1984). 